\title{
DRIVER AND DRIVING ASSESSMENT ISSUES ASSOCIATED WITH THE APPLICATION OF A SECONDARY TASK TECHNIQUE: A CASE STUDY
}

\author{
Michael P. Manser \\ Jacqueline Jenkins \\ Human Factors Program \\ Texas Transportation Institute \\ Texas A\&M University \\ College Station, Texas, USA \\ E-mail: m-manser@ttimail.tamu.edu
}

\begin{abstract}
A review of experimental methodology can uncover the shortcomings of a particular driving assessment technique, providing insight for the development and refinement of this assessment technique, and its application for further studies. By employing a case study as the backdrop for such a review, a context is created within which the specific application of the assessment technique can be examined and discussed. This paper presents the results of the examination of the driver assessment techniques as applied to the case study which investigated the relationship between conversation intensity while using a cell phone and driver performance. A secondary task technique was applied to study the influence of the intensity of conversation on the degradation of driving performance attributed to using a cellular telephone. Forty participants drove through simulated driving environments and engaged in cellular telephone conversations with the experimenter. After driving through each environment participants provided a rating of the workload experienced during the cellular telephone conversation during the drive. Accelerator input, speed, steering input, and lane position measurements were recorded while participants drove through particular sections of the simulated driving environments. Results of the study indicated the variation of steering input increased when the cellular telephone was used. As far as differences between the male participants and females participants, the males generally drove faster and the females responded more quickly to a situation requiring a sudden braking or steering maneuver. Overall, the drivers perceived the workload to be greater when using a cellular telephone.
\end{abstract}

However, the use of the case study to examine the driver assessment and secondary task techniques indicated there were several positive and negative attributes which need to be considered in future research where such techniques are to be applied. For example, an analysis of the performance measures revealed that changes in the horizontal alignment of the roadway created lasting perturbations in the data. In particular, increased variations in steering input and lane position were observed for a considerable distance and time after a participant exited a curved section of roadway. These perturbations subsequently interfered with the application of the secondary task.

Results of this case study will be discussed in terms of the positive and negative attributes of employing the driving assessment techniques and secondary techniques, how to enhance the utility of these techniques, and the theoretical and practical application of these techniques for on-road and simulated driving environment research. 